\title{
Article \\ An In-House Cone-Beam Tomographic Reconstruction Package for Laboratory X-ray Phase-Contrast Imaging
}

\author{
Jürgen Hofmann (D) and Robert Zboray *
}

Citation: Hofmann, J.; Zboray, R. An In-House Cone-Beam Tomographic Reconstruction Package for Laboratory X-ray Phase-Contrast Imaging. Appl. Sci. 2022, 12, 1430. https://doi.org/10.3390/ app12031430

Academic Editors: Brian Abbey and Benedicta Arhatari

Received: 16 December 2021

Accepted: 26 January 2022

Published: 28 January 2022

Publisher's Note: MDPI stays neutral with regard to jurisdictional claims in published maps and institutional affiliations.

Copyright: (C) 2022 by the authors. Licensee MDPI, Basel, Switzerland. This article is an open access article distributed under the terms and conditions of the Creative Commons Attribution (CC BY) license (https:// creativecommons.org/licenses/by/ $4.0 /)$.
Center for X-ray Analytics, Department Materials Meet Life, Swiss Federal Laboratories for Material Science and Technology, Empa, Überlandstrasse 129, 8600 Dübendorf, Switzerland; juergen.hofmann@empa.ch

* Correspondence: robert.zboray@empa.ch

\begin{abstract}
Phase-contrast, and in general, multi-modal, X-ray micro-tomography is proven to be very useful for low-density, low-attention samples enabling much better contrast than its attenuationbased pendant. Therefore, it is increasingly applied in bio- and life sciences primarily dealing with such samples. Although there is a plethora of literature regarding phase-retrieval algorithms, access to implementations of those algorithms is relatively limited and very few packages combining phaseretrieval methods with the full tomographic reconstruction pipeline are available. This is especially the case for laboratory-based phase-contrast imaging typically featuring cone-beam geometry. We present here an in-house cone-beam tomographic reconstruction package for laboratory X-ray phasecontrast imaging. It covers different phase-contrast techniques and phase retrieval methods. The paper explains their implementation and integration in the filtered back projection chain. Their functionality and efficiency will be demonstrated through applications on a few dedicated samples.
\end{abstract}

Keywords: X-ray tomography; CT; cone-beam CT; phase contrast imaging; dark-field imaging; laboratory setup

\section{Introduction}

Over the last two decades, the capabilities and potentials of X-ray computed tomography (CT) and micro-CT has been greatly improved by applying phase-contrast methods, or in general, multi-contrast imaging modalities (phase-contrast, dark-field imaging). This is especially beneficial for biomedical and life science applications, which typically involve low-attenuation-contrast samples that produce very low contrast-to-noise ratio (CNR) images and inferior quality for conventional, attenuation-based CT. In contrast to conventional $\mathrm{X}$-ray $\mathrm{CT}$, phase-contrast techniques rely on detecting small-angle refraction of the $\mathrm{X}$-ray waves traversing the sample and the related phase shifts imparted by the sample [1-3]. This can be two to three orders of magnitude more sensitive than attenuation contrast in case of soft tissues and bio-samples in the hard X-ray regime. In this way, they extend the capabilities of X-ray imaging to weakly or non-attenuating samples or to those details that lack sufficient visibility in conventional CT. Another interesting modality shedding light on nano-scale structures is X-ray dark field imaging. It relies on ultra-small angle scattering of $\mathrm{X}$-rays in the sample by micro- and nano-scale, typically sub-resolution, structures [4-6]. Reviews and comparison of X-ray phase-contrast imaging techniques are given in Refs. [3,7-11]. Phase-contrast methods were first introduced on synchrotron sources but they found their way relatively fast to laboratory sources. This is due to the developments and availability in micro- and nano-focus $X$-ray sources and X-ray optical elements such as diffraction gratings. The main techniques used for laboratory $\mathrm{X}$-ray phase-contrast imaging (PCI) include grating-based PCI [10,12,13], propagation-based PCI [1,2,14], speckle-based PCI [15] and edge illumination [16].

Although there is a plethora of literature regarding phase-retrieval algorithms, especially for propagation-based PCI, access to implementations of those algorithms is relatively 
limited [17-22]. Some of the packages work following the conventional pipeline and start with a phase retrieval step on the $2 \mathrm{D}$ projection images, which are then back projected using mostly filtered back projection (FBP) or iterative reconstruction methods $[18,19,22]$. It was also shown by Ruhlandt and Salditt [23] that phase retrieval and reconstruction are interchangeable also when considering approaches other than FBP, such as the Algebraic Reconstruction Technique (ART). Another work compared the conventional pipeline with 3D post-reconstruction phase retrieval, which has definitely benefits when projections images have to be stitched to achieve larger field-of-views (FOVs) at high resolution [20].

Only few packages wrap the phase-retrieval module with other codes for the full 3D tomographic reconstruction pipeline [18,19,21,22], which are available in open source form to the community. These, except for [21], which is a toolbox coupled to the ASTRA package [24], are developed exclusively for imaging at synchrotrons in parallel beam geometry. However, for phase-contrast CT using laboratory-based setup with cone-beam geometry, there is a real lack of open source reconstruction packages. Note that much more open source packages are available for conventional, attenuation-based CT reconstruction. The review of these is beyond the scope of the present paper.

Here, we introduce an in-house cone-beam PCI reconstruction package with several options for phase-contrast and dark-field micro-CT reconstruction on laboratory sources. The package enables CT reconstruction for grating-based and propagation-based PCI in cone beam geometries and features a graphical user interface. In the following, we briefly describe which methods and how are implemented in the package. After that, we will give representative examples demonstrating how the package works (see Figures 1-3). Note that the goal of the paper is not to perform a benchmark of our package against others. That would be a major effort requiring among others the definition of a consistent and suitable data set for comparison and that is clearly beyond the scope of this paper.

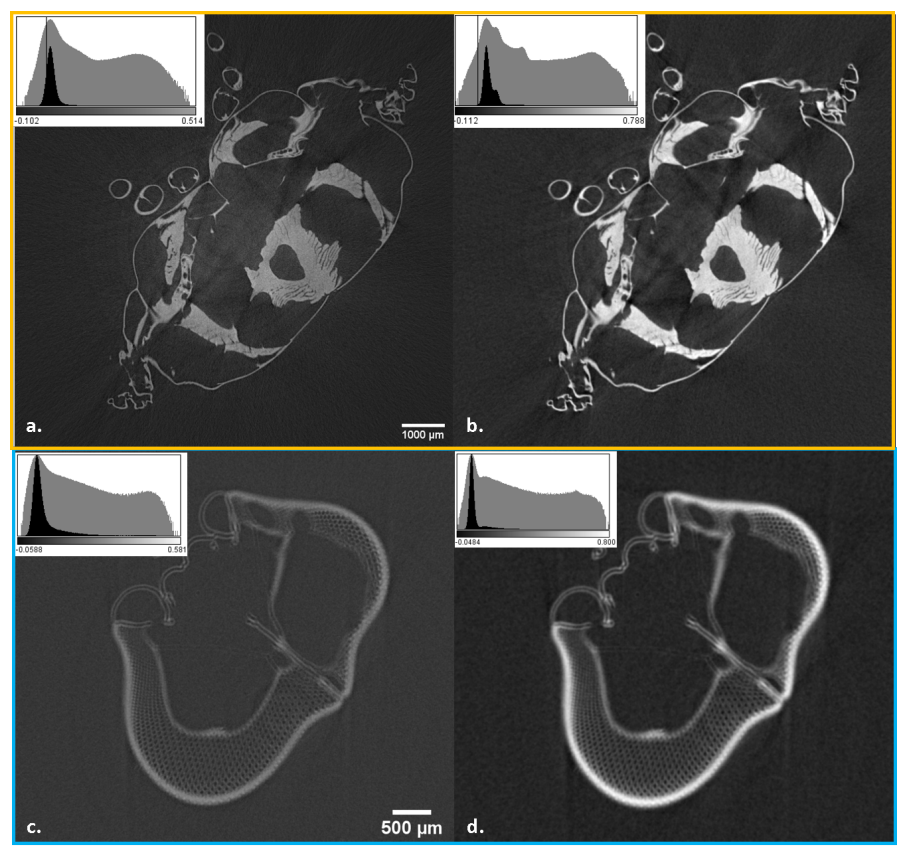

Figure 1. Micro-CT slices of a horse fly based on attenuation $(\mathbf{a}, \mathbf{c})$, and based on Paganin phase retrieval $(\mathbf{b}, \mathbf{d})$. The upper images are showing a horizontal slice at the region of the flight muscles (orange framed in Figure 4a,b), whereas the lower images are taken at the level of the compound eye showing clearly the individual ommatidia (blue framed in Figure 4c,d). The insets show the gray value histograms for the different images (black-linear scale, gray-log scale is shown to be able to better discern the differences). It is clear from those that the Paganin method strongly increases the CNR of the images as the histograms are becoming much more structured, showing several peaks compared to the attenuation case. A $v$ value of 10.0 was used in Equation (6) to obtain the images. The CNR values for the different methods and samples are summarized in Table 1. 

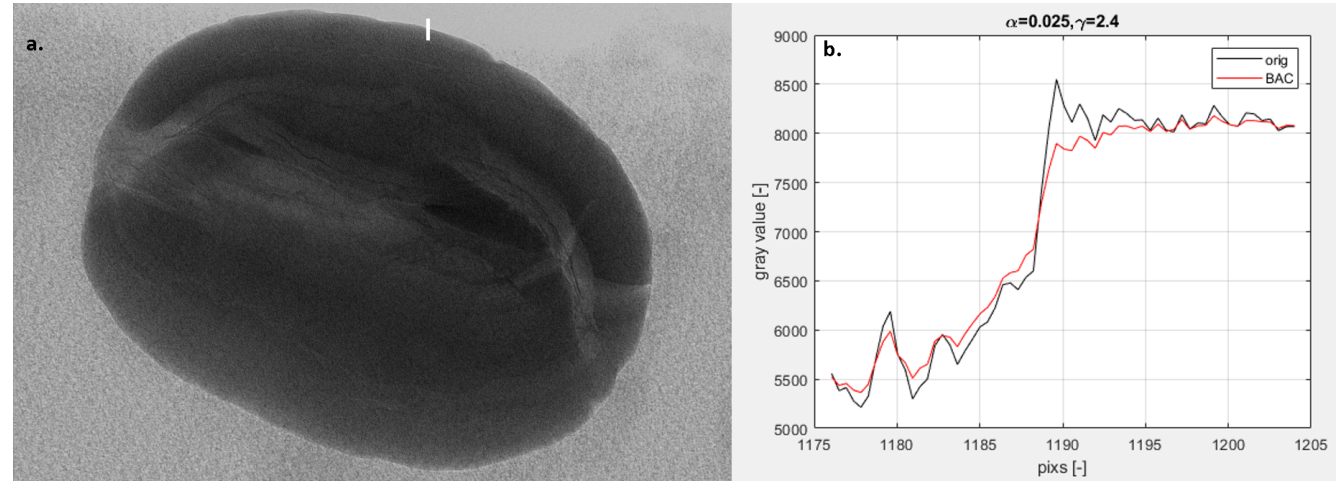

Figure 2. (a) Propagation based projection image of a coffee bean (b) Illustrates the result of the optimization of the $\alpha$ and $\gamma$ parameters of the BAC method showing a comparison of the original and BAC corrected image gray scale profiles over the white vertical line shown in (a). Note that foamy looking material around the bean is florist's foam in which the bean was embedded for the scan.

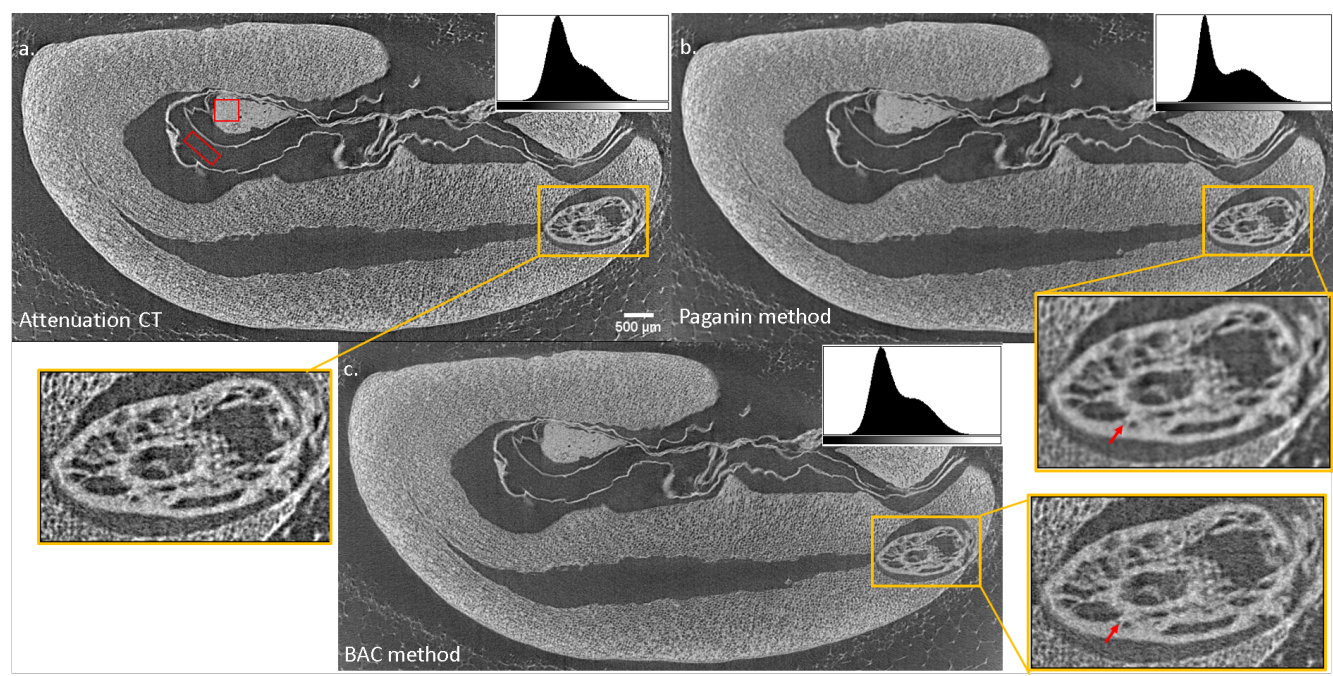

Figure 3. (a) Attenuation-based micro-CT image of a coffee bean. Propagation-based micro-CT images of the coffee bean reconstructed by (b) Paganin's phase retrieval method, Equation (6), and (c) by the BAC method Equations (3) and (4). Note the foamy looking material around the bean is florist's foam in which the bean was embedded for the scan. The insets in the upper right corner of each image show the gray value histograms. It shows that the separation, i.e., CNR increases for the BAC and Paganin retrieval compared to the attenuation case. The regions of interest outlined in orange show with higher magnification how the different retrievals might blur the visibility of small-scale feature (see, e.g., red arrows). For the BAC method the optimized parameters from Figure 2b, whereas for the Paganin method $v=10.0$ were used. The areas circumvented in red indicate the ROIs used for determining the CNR values between sample material and air (see the values in Table 1).

\section{Materials and Methods}

A detailed description of our cone-beam CT reconstruction package for attenuation imaging can be found elsewhere [25]. It describes the technical implementation and GPUbased acceleration of the FDK algorithm for cone-beam reconstruction [26] together with the full data processing workflow from preprocessing, artefact corrections, center of rotation correction to reconstruction. Furthermore, we evaluated the performance of the basic FDK backprojection chain for typical, large micro-CT data sets benchmarking for different computational system architectures [25]. We describe here only the methods implemented in our phase-contrast CT reconstruction modules and how they are interface to the rest. The description is divided according to the two major PCI techniques that are implemented in the package. 


\subsection{Propagation-Based PCI}

A simple formulation for the normalized, propagated intensity at the detector can be derived based on the so-called transport-of-intensity (TIE) equation [2,3,27]:

$$
\frac{I(\mathbf{r}, z)}{I(\mathbf{r}, 0)}=1-\frac{z}{k} \nabla^{2} \phi(\mathbf{r})
$$

where $z$ is the propagation distance, $k=2 \pi / \lambda$ is the wave-number and $\phi(\mathbf{r})$ is the phase shift inflicted by the sample. The propagation distance in Equation (1) has to be replaced by $z_{\text {eff }}=z / M$ for cone-beam geometry and $\mathbf{r}$, representing the detection plane coordinates perpendicular to the optical axis, has to be also scaled by $M$, the magnification, according to the Fresnel scaling theorem [2]. The TIE gives satisfactory results in the so-called direct contrast regime, i.e., for moderate propagation distances and for most practical cases for laboratory PCI. The simplest form of TIE-based phase retrieval is obtained by inversion of Equation (1) via Fourier transform $(\mathcal{F})$ as

$$
\phi(\mathbf{r})=-\frac{k}{z} \mathcal{F}^{-\infty}\left[\frac{\mathcal{F}[I(\mathbf{r}, z) / I(\mathbf{r}, 0)-1]}{\mathbf{k}^{2}}\right]
$$

$\mathbf{k}$ is the Fourier-space equivalent of $\mathbf{r}$. Equation (2) is the phase retrieval step of the original Bronnikov algorithm [28], which was integrated together with the ramp filter into the tomographic reconstruction. As explained above, the phase retrieval can be also performed prior to reconstruction on the projection images as is done mostly in practice, which is also implemented in our reconstruction package. The contact image $\mathrm{I}(\mathbf{r}, 0)$ is experimentally challenging and not practical to measure. For pure phase objects or negligible attenuation it represents a normalization by an open-beam (no object) image, $I_{0}$, instead of $I(\mathbf{r}, 0)$ in Equation (2). Finite object attenuation is typically taken into account adding an empirical regularization parameter, $\alpha$, to the denominator of Equation (2) also to compensate the singularity at zero frequency $(\mathbf{k}=0)$ and stabilize the reconstruction. This leads to:

$$
\phi(\mathbf{r})=-\frac{k}{z} \mathcal{F}^{-1}\left[\frac{\mathcal{F}\left[I(\mathbf{r}, z) / I_{o}-1\right]}{\alpha+\mathbf{k}^{2}}\right]
$$

Equation (3) is the modified Bronnikov algorithm (MBA) [29]. De Witte et al. [30] proposed an alternative approach the so-called Bronnikov-Aided-Correction (BAC), which is a two-step algorithm. In the first step, an approximation for the phase distribution, $\phi(\mathbf{r})$ is obtained by applying Equation (3). Then the contact image is estimated by expressing it with the help of Equation (1) as

$$
I(\mathbf{r}, 0)=\frac{I(\mathbf{r}, z)}{1-\gamma \nabla^{2} \phi(\mathbf{r})}
$$

where $z / k$ was replaced by a control parameter $\gamma$ that is typically determined empirically together with $\alpha$ [2]. A common strategy for determining $\alpha$ is to examine in $\phi(\mathbf{r})$ obtained in the first step profiles through edges and material boundaries and decrease $\alpha$ starting from larger values until edge effects are eliminated. Then adjust the strength of the phase correction in the second step increasing $\gamma$ starting from small values and its final value is determined by visual inspection. We also apply this strategy with our reconstruction package and enable the user to choose the $\alpha$ and $\gamma$ parameters (see an illustration of it in Figures 2 and 4).Then the contact image, $I(\mathbf{r}, 0)$, is used further in the conventional attenuation CT reconstruction pipeline. 
Besides the BAC algorithm, we implemented in our package the likely most often applied single-distance phase retrieval algorithm by Paganin et al. [31]. It is derived assuming a fixed-stoichiometry object, i.e., with a constant $\delta / \beta$ ratio, as

$$
\phi(\mathbf{r})=-\frac{\delta}{2 \beta} \ln \left(\mathcal{F}^{-1}\left[\frac{\mathcal{F}\left\{I(\mathbf{r}, z) / I_{o}\right\}}{1+\frac{\lambda z \delta}{4 \pi \beta}|\mathbf{k}|^{2}}\right]\right)
$$

where $\delta$ is the decrement from one of the real part, $\beta$ is the imaginary part of the complex index of refraction of the sample at the given wave length $(n=1-\delta(\lambda)+i \beta(\lambda))$. This is in effect very similar to the MBA algorithm representing a second-order low-pass filter in the frequency domain. For laboratory propagation-based PCI, where one has a broad energy spectrum, typically an effective wave length is chosen in the denominator of Equation (4) mostly based on visual examination of the retrieved phase image similar to the choice of the $\alpha$ parameter of the MBA method. Therefore, we implemented it by lumping the coefficient in front of $|\mathbf{k}|^{2}$ into a single, user-tunable parameter, $v$, in a slightly modified form as a preprocessing image (Lorentzian) filter as discussed in [32]:

$$
I_{f i l}(\mathbf{r}, z)=\mathcal{F}^{-1}\left[\frac{\mathcal{F}\{I(\mathbf{r}, z)\}}{1+v|\mathbf{k}|^{2}}\right]
$$

$I_{f i l}$ is then used in the conventional cone-beam reconstruction pipeline.

\subsection{Grating-Based PCI}

Typical laboratory grating-based PCI setup feature typically a high-power, macro-focus laboratory X-ray source and a G0 source gratings to increase the spatial coherence of the radiation and the usual G1 and G2 gratings forming the Talbot-Lau interferometer $[10,12,13,33]$. To obtain the attenuation, the differential phase-contrast and dark-field images simultaneously, mostly a phase-stepping approach, usually moving the G1 grating cross-wise in small, equidistant steps is used on such Talbot-Lau setup. The Fourier decomposition of the phase stepping curve approximates it up to the second term as:

$$
I(\mathbf{r}, \chi)=a_{0}+a_{1} \sin \left(\frac{2 \pi \chi}{p}+\phi\right)
$$

where $\chi$ is the phase step position and $p$ is the grating period of the grating being stepped. This is a sound assumption for rectangular gratings of a reasonable quality. It requires that the phase stepping corresponds to one or several full periods of the grating that is analysed. The mean and amplitude and phase factors in Equation (7) are obtained by pixel-wise fast Fourier transform (FFT) of the stepping images. Then three image modalities are defined as:

$$
\begin{aligned}
A(\mathbf{r}) & =\frac{a_{0, s}}{a_{0, r e f}} \\
\operatorname{DPC}(\mathbf{r}) & =\phi_{r e f}-\phi_{s} \\
\operatorname{DFI}(\mathbf{r}) & =\frac{a_{1, s} a_{0, r e f}}{a_{1, r e f} a_{0, s}}
\end{aligned}
$$

where $A, D P C$ and $D F I$ are the attenuation, differential phase contrast and dark-field projection images, respectively and the subscripts ref and $s$ are for empty beam reference and for sample images. To obtain the CT images of $\delta$ of the sample from the DPC images, instead of the usual apodization filters, such as Ram-Lak and Shepp-Logan filters [25] for attenuation CT, a Hilbert-filter is used [10]. Note that for reconstructing both the the phase-contrast and dark-field CT, the option of taking no logarithm of normalised images is built in the package. 
Besides the Fourier decomposition, we have also implemented a direct least-squares fit to determine the Fourier components in Equation (7) [33]. In contrast to the Fourier method this method does not need equidistant sampling of the phase stepping curve in Equation (7) in $[0,2 \pi]$. For this, Equation (7) is linearized as

$$
I(\mathbf{r}, \chi)=a_{0}+b_{1} \sin \left(\frac{2 \pi \chi}{p}\right)+b_{2} \cos \left(\frac{2 \pi \chi}{p}\right)
$$

where

$$
a_{1}=\sqrt{b_{1}^{2}+b_{2}^{2}}
$$

and

$$
\phi=\frac{b_{2}}{b_{1}}
$$

The three parameter $a_{0, i}, b_{1, i}, b_{2, i}$ for pixel $i$ are efficiently determined by least-square matrix inversion using the Penrose pseudo inverse, e.g., in MATLAB. Both methods deliver very similar results in general as is shown in Kaufmann et al. [33].

\section{Results and Discussion}

This section discusses some examples demonstrating the functioning of our in-house reconstruction package for different types of phase-contrast micro-CT methods.

\subsection{Example for the Paganin Method}

To demonstrate the functioning of our in-house cone-beam tomographic reconstruction package for laboratory phase-contrast imaging using the Paganin phase-retrieval technique, we will use as an example the propagation-based micro-CT of a horse fly. The scan was performed on an EasyTom XL Ultra 230-160 micro/nano-CT scanner (RxSolutions SAS, Chavanod, France). The scanner features a Hamamatsu reflection-type micro-focus X-ray tube. The scan was performed using a MH110XC-KK-FA, XiRay detector from XIMEA that features a 11 MPixel CCD chip coupled directly via an optical fibre bundle to a $20 \mu \mathrm{m}$ thick GadOx scintillator and having a native pixel size of $9 \mu \mathrm{m}$ and 14 bits of dynamic range. The tube was operated at $65 \mathrm{kVp}$ and a current of $120 \mu \mathrm{A}$, i.e., at slightly lower than $8 \mathrm{~W}$ tube power rendering an emission spot size of around $4 \mu \mathrm{m}$. The geometrical magnification was 1.16 and the voxel size of the CT scan was $7.8 \mu \mathrm{m}$. Thus, the setup represents a so-called inverse geometry with relatively short propagation distance and a high resolution detector as opposed to the traditional large propagation-distance setup with a large pixel size detector. The images were acquired with 4.0 s exposure time per projection. 1600 projections were taken over 360 degree sample rotation. Results are given in Figure 1 showing the cross-sectional CT slices at two regions: one where the flight muscles are located (see orange line in Figure 4a) and one at the level of the compound eyes (see blue line in Figure 4a). The latter clearly resolves the individual ommatidia. From the images and the corresponding gray-scale histograms, it is clear that the Paganin method strongly increases the CNR of the images and correspondingly the histograms are becoming much more structured, showing several peaks compared to the attenuation case. This means that the different structures corresponding to the different gray-scale peaks can be better resolved in the phase image. This is a well-known property of the Paganin method and the corresponding filter $[34,35]$. The CNR values for the different methods and samples are summarized in Table 1. A $v$ value of 10.0 was used in Equation (6) to obtain the images. For a projection image of the horse fly see Figure $4 \mathrm{a}$. 

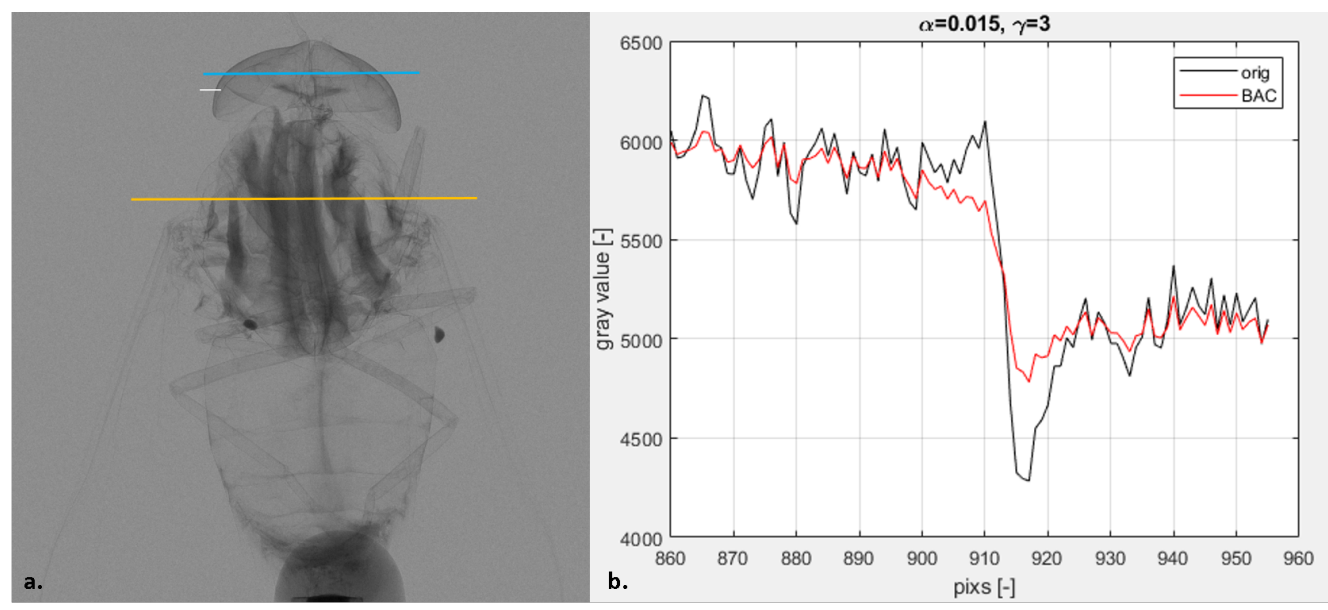

Figure 4. (a) Propagation based projection image of the horse fly. (b) Illustration of the result of the optimization of the $\alpha$ and $\gamma$ parameters of the BAC method showing a comparison of the original and BAC corrected image gray scale profiles over the white horizontal line shown in (a).

Table 1. CNR values between sample material and air for the different $\mathrm{CT}$ images and reconstruction methods. For the ROIs used to determine the CNR see Figures 3 and 5.

\begin{tabular}{cccc}
\hline Sample & Attenuation CT & Paganin Method & BAC Method \\
\hline horse fly & 7.5 & 17.7 & 10.8 \\
coffe bean & 2.8 & 7.0 & 3.4 \\
\hline
\end{tabular}

\subsection{Example BAC Method}

In this subsection, we demonstrate the implementation of the BAC method in our in-house reconstruction package and compare to the Paganin method, using a propagationbased micro-CT of a coffee bean. The scan was performed using the same scanner as in the previous subsection. The scan parameter were slightly different: the tube was operated at $65 \mathrm{kVp}$ and a current of $150 \mu \mathrm{A}$.The geometrical magnification was 2.53 and the voxel size of the CT scan was $7.1 \mu \mathrm{m}$. The CCD camera was used with $2 \times 2$ pixel binning. The images were acquired with $4.0 \mathrm{~s}$ exposure time per projection. 1568 projections were taken over 360 degree sample rotation. Figure $2 \mathrm{a}$ shows a projection image of the coffee bean and in Figure $2 \mathrm{~b}$ shows the result of the optimization process for the $\alpha$ and $\gamma$ parameters as is explained in the previous section. Micro-CT images of the coffee bean are given in Figure 3. These show that the separation in the gray-value histogram increases, i.e., both the Paganin and BAC methods increase the CNR of the reconstructed images (see Table 1 for actual values). Furthermore, the magnified regions of interest (ROIs) framed in orange show how the different retrievals might blur the visibility of small scale feature (see, e.g., red arrows). The BAC method, as it has been reported earlier $[29,30,36]$, improves significantly the CNR compared to the attenuation-based image but, in general, blurs less the images than the Paganin method and therefore enables resolving very tiny features (see red arrows in Figure 3).

Finally, we compare the attenuation-based, Paganin phase retrieval and BAC methods for the horse fly sample from the previous subsection as well. This is shown in Figure 5.

\subsection{Example for Grating-Based PCI}

In this paragraph, we show the functioning of our in-house cone-beam tomographic reconstruction package for laboratory phase-contrast imaging using the grating based technique as described in Section 2.2. The sample was a very-low-attenuation, porous cellulose foam sample. The scan was performed using our in-house grating-based PCI micro-CT setup. Technical details of the setup are given in [33]. The scan was performed at $40 \mathrm{kVp}$ tube voltage and a tube current of $20 \mathrm{~mA}$. We performed the scan with seven 
phase steps per projection each with $2.5 \mathrm{~s}$ exposure time. We took overall 360 projections over 360 degree sample rotation. The geometrical magnification of the setup is very low 1.17. Figure 6 shows some illustrative results proving the power of PCI and DFI for lowattenuation, porous samples. The attenuation image is so noisy due to the very low sample attenuation that the internal porous structure of the sample cannot be discerned. Only the phase-contrast and the dark-field CT images show these structures. They nicely illustrate inverse gray scale values with respect to each other as expected: pores are shown with low intensity in the PCI, whereas they have high intensities in the DFI and vice versa for the cellulose material.
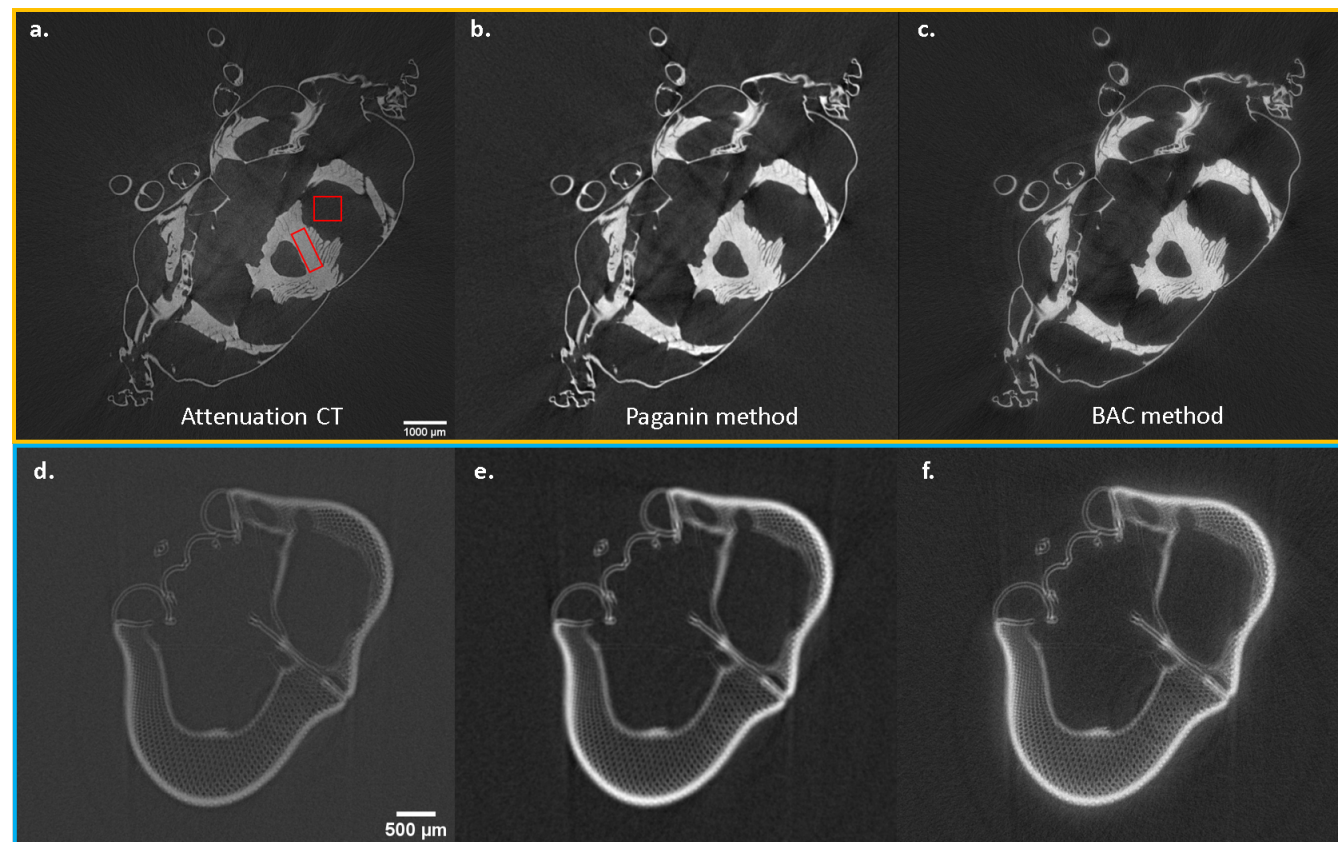

\section{f.}

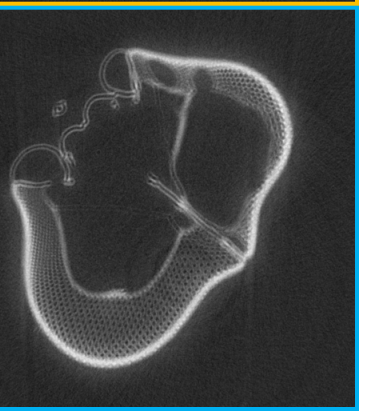

Figure 5. Comparison of the attenuation-based micro-CT images of the horse fly $(\mathbf{a}, \mathbf{d})$, with the propagation-based images reconstructed by using Paganin's phase retrieval method, (b,e), based on Equation (6), and with the BAC method based on Equations (3) and (4), (c,f). The upper images show a horizontal slice at the region of the flight muscles (see orange line in Figure 4a), whereas the lower images are taken at the level of the compound eye showing clearly the individual ommatidia (see the blue line in Figure 4a). The areas circumvented in red indicate the ROIs used for determining the CNR values between sample material and air (see Table 1).
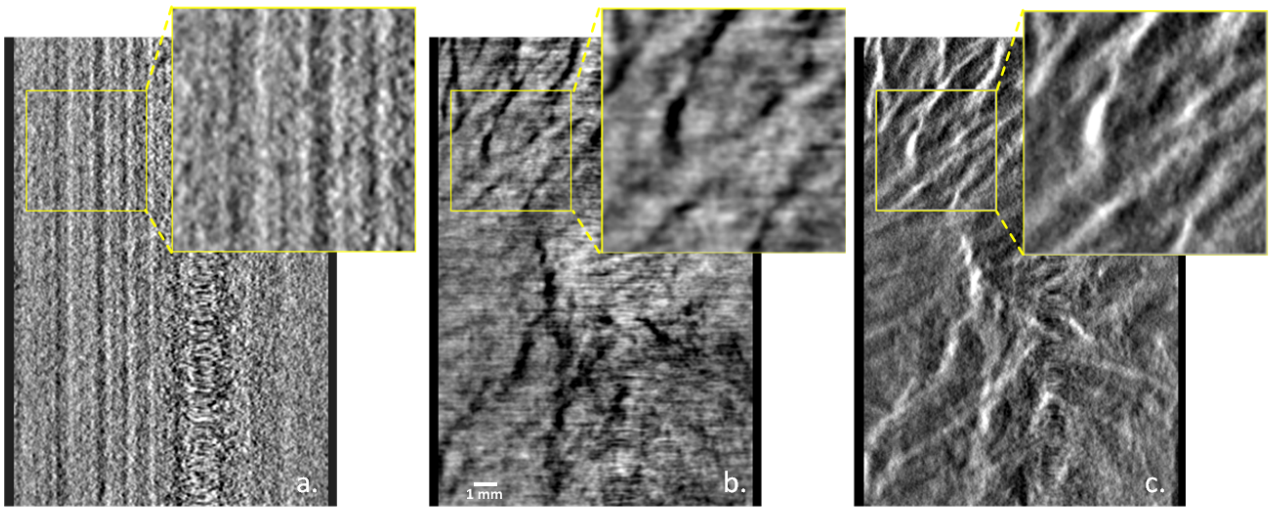

Figure 6. Grating based phase contrast tomogram slice of a cellulose foam. (a) shows the attenuation, (b) the phase contrast and (c) the dark field, small angle scattering, image. 


\section{Conclusions}

We introduce and describe an in-house, cone-beam tomographic reconstruction package for laboratory phase-contrast imaging. The package covers different phase-contrast techniques and phase retrieval methods. It includes the well-known Paganin method and the Bronnikov-aided Correction method for propagation-based phase-contrast imaging. It also includes reconstruction method for the grating-based phase-contrast and dark-field micro-CT imaging. In the paper, we explain the implementation of these methods and their integration in the filtered back projection pipeline. Their functionality and efficiency were demonstrated by performing phase-contrast micro-CT on a few dedicated bio-samples. The source code of the package is published as open source under the GNU Lesser General Public License Version 3 at the repository at https:/ / github.com/JueHo/CT-Recon (accessed on 1 October 2020). The authors welcome usage of and contributions to the package by the community. Please contact them for more information or go directly to the above project repository.

Author Contributions: Conceptualization, J.H. and R.Z.; methodology, J.H. and R.Z.; software, J.H.; validation, J.H. and R.Z.; formal analysis, J.H. and R.Z.; investigation, J.H. and R.Z.; writing—original draft preparation, J.H. and R.Z.; writing-review and editing, J.H. and R.Z. All authors have read and agreed to the published version of the manuscript.

Funding: This research received no external funding.

Institutional Review Board Statement: Not applicable.

Informed Consent Statement: Not applicable.

Data Availability Statement: Not applicable.

Conflicts of Interest: The authors declare no conflict of interest.

\section{Abbreviations}

The following abbreviations are used in this manuscript:

$\begin{array}{ll}\text { ART } & \text { algebraic reconstruction technique } \\ \text { BAC } & \text { Bronnikov-aided correction } \\ \text { CCD } & \text { charge-coupled device } \\ \text { CNR } & \text { contrast-to-noise ratio } \\ \text { CT } & \text { computed tomography } \\ \text { DFI } & \text { dark-field image } \\ \text { DPC } & \text { differential phase contrast } \\ \text { FBP } & \text { filtered back projection } \\ \text { FDK } & \text { Feldkamp-Davis-Kress algorithm } \\ \text { FFT } & \text { Fast Fourier Transform } \\ \text { GPU } & \text { graphics processing unit } \\ \text { MBA } & \text { modified Bronnikov algorithm } \\ \text { PCI } & \text { phase-contrast imaging } \\ \text { ROI } & \text { region of interest } \\ \text { TIE } & \text { transport of intensity equation }\end{array}$

\section{References}

1. Pogany, A.; Gao, D.; Wilkins, S. Contrast and resolution in imaging with a microfocus X-ray source. Rev. Sci. Instrum. 1997, 68, 2774-2782. [CrossRef]

2. Salditt, T.; Aspelmeier, T.; Aeffner, S. Biomedical Imaging: Principles of Radiography, Tomography and Medical Physics; Walter de Gruyter GmbH \& Co. KG: Berlin, Germany, 2017.

3. Endrizzi, M. X-ray phase-contrast imaging. Nucl. Instrum. Methods Phys. Res. Sect. A Accel. Spectrom. Detect. Assoc. Equip. 2018, 878, 88-98. [CrossRef]

4. Pfeiffer, F.; Bech, M.; Bunk, O.; Kraft, P.; Eikenberry, E.F.; Brönnimann, C.; Grünzweig, C.; David, C. Hard-X-ray dark-field imaging using a grating interferometer. Nat. Mater. 2008, 7, 134-137. [CrossRef] [PubMed] 
5. Yashiro, W.; Terui, Y.; Kawabata, K.; Momose, A. On the origin of visibility contrast in X-ray Talbot interferometry. Opt. Express 2010, 18, 16890-16901. [CrossRef] [PubMed]

6. Strobl, M. General solution for quantitative dark-field contrast imaging with grating interferometers. Sci. Rep. 2014, 4, 7243. [CrossRef] [PubMed]

7. Cloetens, P.; Barrett, R.; Baruchel, J.; Guigay, J.P.; Schlenker, M. Phase objects in synchrotron radiation hard X-ray imaging. J. Phys. D Appl. Phys. 1996, 29, 133. [CrossRef]

8. Wilkins, S.; Gureyev, T.E.; Gao, D.; Pogany, A.; Stevenson, A. Phase-contrast imaging using polychromatic hard X-rays. Nature 1996, 384, 335-338. [CrossRef]

9. Momose, A.; Kawamoto, S.; Koyama, I.; Hamaishi, Y.; Takai, K.; Suzuki, Y. Demonstration of X-ray Talbot interferometry. Jpn. J. Appl. Phys. 2003, 42, L866. [CrossRef]

10. Pfeiffer, F.; Weitkamp, T.; Bunk, O.; David, C. Phase retrieval and differential phase-contrast imaging with low-brilliance X-ray sources. Nat. Phys. 2006, 2, 258-261. [CrossRef]

11. Diemoz, P.; Bravin, A.; Coan, P. Theoretical comparison of three X-ray phase-contrast imaging techniques: Propagation-based imaging, analyzer-based imaging and grating interferometry. Opt. Express 2012, 20, 2789-2805. [CrossRef]

12. Bech, M.; Jensen, T.H.; Feidenhans, R.; Bunk, O.; David, C.; Pfeiffer, F. Soft-tissue phase-contrast tomography with an X-ray tube source. Phys. Med. Biol. 2009, 54, 2747. [CrossRef] [PubMed]

13. Pfeiffer, F.; Bunk, O.; Kottler, C.; David, C. Tomographic reconstruction of three-dimensional objects from hard X-ray differential phase contrast projection images. Nucl. Instrum. Methods Phys. Res. Sect. A Accel. Spectrom. Detect. Assoc. Equip. 2007, 580, 925-928. [CrossRef]

14. Mayo, S.C.; Stevenson, A.W.; Wilkins, S.W. In-line phase-contrast X-ray imaging and tomography for materials science. Materials 2012, 5, 937-965. [CrossRef]

15. Zdora, M.C. State of the art of X-ray speckle-based phase-contrast and dark-field imaging. J. Imaging 2018, 4, 60. [CrossRef]

16. Zamir, A.; Hagen, C.; Diemoz, P.C.; Endrizzi, M.; Vittoria, F.; Chen, Y.; Anastasio, M.A.; Olivo, A. Recent advances in edge illumination X-ray phase-contrast tomography. J. Med. Imaging 2017, 4, 040901. [CrossRef] [PubMed]

17. Weitkamp, T.; Haas, D.; Wegrzynek, D.; Rack, A. ANKAphase: Software for single-distance phase retrieval from inline X-ray phase-contrast radiographs. J. Synchrotron Radiat. 2011, 18, 617-629. [CrossRef] [PubMed]

18. Gürsoy, D.; De Carlo, F.; Xiao, X.; Jacobsen, C. TomoPy: A framework for the analysis of synchrotron tomographic data. J. Synchrotron Radiat. 2014, 21, 1188-1193. [CrossRef]

19. Mirone, A.; Brun, E.; Gouillart, E.; Tafforeau, P.; Kieffer, J. The PyHST2 hybrid distributed code for high speed tomographic reconstruction with iterative reconstruction and a priori knowledge capabilities. Nucl. Instrum. Methods Phys. Res. Sect. B Beam Interact. Mater. Atoms 2014, 324, 41-48. [CrossRef]

20. Brun, F.; Brombal, L.; Di Trapani, V.; Delogu, P.; Donato, S.; Dreossi, D.; Rigon, L.; Longo, R. Post-reconstruction 3D single-distance phase retrieval for multi-stage phase-contrast tomography with photon-counting detectors. J. Synchrotron Radiat. 2019, 26, 510-516. [CrossRef]

21. Lohse, L.M.; Robisch, A.L.; Töpperwien, M.; Maretzke, S.; Krenkel, M.; Hagemann, J.; Salditt, T. A phase-retrieval toolbox for X-ray holography and tomography. J. Synchrotron Radiat. 2020, 27, 852-859. [CrossRef]

22. Langer, M.; Zhang, Y.; Figueirinhas, D.; Forien, J.B.; Mom, K.; Mouton, C.; Mokso, R.; Villanueva-Perez, P. PyPhase-A Python package for X-ray phase imaging. J. Synchrotron Radiat. 2021, 28, 1261-1266. [CrossRef] [PubMed]

23. Ruhlandt, A.; Salditt, T. Three-dimensional propagation in near-field tomographic X-ray phase retrieval. Acta Crystallogr. Sect. A Found. Adv. 2016, 72, 215-221. [CrossRef] [PubMed]

24. The ASTRA Toolbox. Available online: https://www.astra-toolbox.com/ (accessed on 1 October 2020).

25. Hofmann, J.; Flisch, A.; Zboray, R. Principles for an Implementation of a Complete CT Reconstruction Tool Chain for Arbitrary Sized Data Sets and its GPU Optimization. J. Imaging 2022, 8, 12. [CrossRef] [PubMed]

26. Feldkamp, L.A.; Davis, L.C.; Kress, J.W. Practical cone-beam algorithm. J. Opt. Soc. Am. A 1984, 1, 612-619. [CrossRef]

27. Teague, M.R. Deterministic phase retrieval: A Green's function solution. J. Opt. Soc. Am. 1983, 73, 1434-1441. [CrossRef]

28. Bronnikov, A.V. Reconstruction formulas in phase-contrast tomography. Opt. Commun. 1999, 171, 239-244. [CrossRef]

29. Boone, M.; De Witte, Y.; Dierick, M.; Van den Bulcke, J.; Vlassenbroeck, J.; Van Hoorebeke, L. Practical use of the modified Bronnikov algorithm in micro-CT. Nucl. Instrum. Methods Phys. Res. Sect. B Beam Interact. Mater. Atoms 2009, 267, $1182-1186$. [CrossRef]

30. De Witte, Y.; Boone, M.; Vlassenbroeck, J.; Dierick, M.; Van Hoorebeke, L. Bronnikov-aided correction for X-ray computed tomography. J. Opt. Soc. Am. A 2009, 26, 890-894. [CrossRef] [PubMed]

31. Paganin, D.; Mayo, S.C.; Gureyev, T.E.; Miller, P.R.; Wilkins, S.W. Simultaneous phase and amplitude extraction from a single defocused image of a homogeneous object. J. Microsc. 2002, 206, 33-40. [CrossRef]

32. Carrel, M.; Beltran, M.A.; Morales, V.L.; Derlon, N.; Morgenroth, E.; Kaufmann, R.; Holzner, M. Biofilm imaging in porous media by laboratory X-Ray tomography: Combining a non-destructive contrast agent with propagation-based phase-contrast imaging tools. PLoS ONE 2017, 12, e0180374. [CrossRef]

33. Kaufmann, R.; Plamondon, M.; Hofmann, J.; Neels, A. Comparison of different phase retrieval algorithms. In Developments in X-ray Tomography XI; International Society for Optics and Photonics: Bellingham, WA, USA, 2017; Volume 10391, p. 1039115. 
34. Beltran, M.; Paganin, D.M.; Siu, K.; Fouras, A.; Hooper, S.; Reser, D.; Kitchen, M. Interface-specific X-ray phase retrieval tomography of complex biological organs. Phys. Med. Biol. 2011, 56, 7353. [CrossRef] [PubMed]

35. Kitchen, M.J.; Buckley, G.A.; Gureyev, T.E.; Wallace, M.J.; Andres-Thio, N.; Uesugi, K.; Yagi, N.; Hooper, S.B. CT dose reduction factors in the thousands using X-ray phase contrast. Sci. Rep. 2017, 7, 15953. [CrossRef] [PubMed]

36. Zboray, R. Optimizing and applying high-resolution, in-line laboratory phase-contrast X-ray imaging for low-density material samples. J. Microsc. 2021, 282, 123-135. [CrossRef] [PubMed] 